\title{
correspondence
}

\section{Journal guidelines}

SIR,--In your issue of November 27, you ask for comments on "Journal guidelines", a discussion which you began in your issue of November 6 . You may be interested in publishing the experience with our new journal, Intervirology, which is owned and operated by the Virology Section of the International Society for Microbiological Societies.

The journal has 12 sections, each with its own editor and specialised board of reviewers, who are listed in each issue. For the first five volumes, each author selected the appropriate section of the journal and submitted one copy of the paper to the Section Editor and a second copy to a board reviewer of his choice. In this manner, the author was confident that his paper was being judged by persons whom he regarded as authorities in the area of the work being presented. 'The board member sent his review to the Section Editor, who also reviewed the paper and then made the decision to accept, modify, reject, or seek additional, anonymous review. The final judgement as to publication was made by the Section Editor.

At the Third International Congress for Virology held in Madrid in September, it was decided to modify this procedure. To provide a consistent balance between known and anonymous reviewers, authors are now required to send one copy of their paper to an editorial board member. Essentially, the reviewer acts as the friend in court whom the author respects as an authority in the field. The author also sends two copies to the Section Editor, who reads one copy as before, but who now regularly sends out a copy for anonymous review. As before, reviews are sent to the Section Editor, who continues to make the final judgement on publication.

The editors hope that the current procedure will satisfy authors that they are fairly represented in the decisionmaking process and at the same time will allow for non-personal critical evaluation before a paper is accepted or rejected.

Yours faithfully, JOSEPH L. MELNICK (Editor-in-Chief)

Department of Virology,

Baylor College of Medicine,

Texas 77025
SIR, - lt is extraordinarily difficult to prove the functional value of a complex and subtle social convention such as the preservation of the anonymity of editorial referees. What, for example, is the nature of the 'editorial experience' that Dr C. N. Davies claims (December 18) to confirm his opinion that this long-established custom can be abandoned? His experiment with the Journal of Aerosol Science is to be commended; but we shall need to know more about the results before accepting his personal opinion that they are convincing.

Until such empirical evidence is available, I cling rather to the conventional wisdom that anonymity protects the referee from the temptation of softening his remarks to avoid causing personal offence. Most scientists are intellectually conscientious, and will exercise their critical faculties fairly on any paper within their competence; they are seldom (if ever, in my experience) malicious or dishonest. But they know that the delicate competitive/ cooperative relationship between colleagues in the same 'Invisible College' cannot stand the stresses that would arise if Dr A had to express, in his own name, in writing, his opinion of the earnest efforts of his rival/friend Dr B. Inevitably, punches would be pulled, and fundamental critical issues would be ducked.

Anonymity is better for all concerned: for the referee, who does not have to mix emotional factors into his intellectual judgements; for the editor, who gets a more honest opinion to guide his decisions; for the reader, who gets more reliable and better expressed papers that have been subjected to a higher standard of criticism; and, strangely enough, for the author who, when his mistakes are pointed out, can vent his chagrin harmlessly in the direction of an impersonal critic without falling into the mortal sin of acquiring a supposed enemy.

The cry against 'authoritarianism' and 'elitism' is, of course, populist in that it appeals to the immediate interest of each individual against the social constraints imposed by the community. But the 'anonymous referee' is only oneself, on the other side of the hill, wearing another uniform. Merton and Zuckermann (Minerva, 9, 66; 1971) have found no abuse of the referee system by senior scientists-or whoever the bogies are supposed to be. My own experience is that the one-way mirror of anonymity facilitates a psychological role reversal, from author to referee, which makes better, more humble, more sceptical scientists of us all, and curbs the vanity and pride of those who claim 'authority'.

This, somewhat schematically and cryptically, is the basis of my assertion that it is a populist folly to disclose the names of referees to authors. Yours faithfully, JOHN ZIMAN

University of Bristol, UK

\section{Unit proposal}

SrR,-We would like to propose a unit for electrophoretic mobility $(u)$ to replace the current practice of specifying it, for example, as follows: $u=-2.45 \times 10^{-5} \mathrm{~cm} \mathrm{~s}^{-1} / \mathrm{V} \mathrm{cm}^{-1}$ ( + for cations, - for anions).

In honour of Arne Tiselius, who has done more than anyone else to advance electrophoretic methodology, we propose the adoption of the Tiselius Unit: $1 \mathrm{TU}=10^{-5} \mathrm{~cm} \mathrm{~s}^{-1} / \mathrm{V} \mathrm{cm}^{-1}$ as the unit of the electrophoretic mobility. The above example for electrophoretic mobility will thus be written: $u=-2.45 \mathrm{TU}$ in the proposed notation.

This recommendation relies on analogy to the adopted Svedberg Unit S for sedimentation coefficients $(s)$ : $1 \mathrm{~S}=10^{-13} \mathrm{~s}$, where the sedimentation coefficient

$$
s=\frac{\mathrm{d} r / \mathrm{d} t}{\omega^{2} r}
$$

measures the sedimentation velocity of a particle in a unit centrifugal field, by analogy with the electrophoretic mobility $u$ measuring the electrophoretic velocity in a unit electrical field.

We will now adopt the Tiselius Unit in our publications and urge others to do so, not only to avoid the wastefulness of the present notation, but also to commemorate the scientist who initiated the far-reaching development of analytical and preparative electrophoretic methodology.

Yours faithfully,

Nicholas Catsimpoolas

Massachusetts Institute of Technology Stelian Huerten

University of Uppsala

Alexander Kolin

University of California,

Los Angeles

Jerker Porath

University of Uppsala,

Sweden 